\title{
Maya Deren et la transfiguration filmique du temps
}

\section{Olivier Salazar-Ferrer}

\section{OpenEdition}

Journals

Édition électronique

URL : https://journals.openedition.org/recherchestravaux/680

DOI : 10.4000/recherchestravaux.680

ISSN : 1969-6434

\section{Éditeur}

UGA Éditions/Université Grenoble Alpes

\section{Édition imprimée}

Date de publication : 1 novembre 2014

Pagination : 89-106

ISBN : $978-2-84310-282-0$

ISSN : 0151-1874

\section{Référence électronique}

Olivier Salazar-Ferrer, « Maya Deren et la transfiguration filmique du temps », Recherches \& Travaux [En ligne], 84 | 2014, mis en ligne le 01 avril 2016, consulté le 29 octobre 2021. URL : http://

journals.openedition.org/recherchestravaux/680 ; DOI : https://doi.org/10.4000/recherchestravaux. 680 
Olivier Salazar-Ferrer

Université de Glasgow

\section{Maya Deren et la transfiguration filmique du temps}

Je tenterai dans cette étude de définir le poétique par une tension entre une fonction désorganisatrice et une fonction créatrice. La première engendre un écart avec ce qu'il n'est pas, par un travail de rupture, de désorganisation des codifications existantes de l'expression, que l'on pourrait appeler un facteur catastrophique, ou "diagramme», au sens deleuzien ${ }^{1}$. La seconde fonction est l'invention de formes conditionnant de nouvelles expériences ("the creation of an experience $\left.{ }^{2} »\right)$ qui s'intégreront de façon émotionnelle à la conscience du spectateur, par opposition à toute autre finalité externe, qu'elle soit divertissement ou connaissance, dans une culture socialement codifiée. L'œuvre théorique et cinématographique de la cinéaste expérimentale Maya Deren (Eleanora Derenkowsky, I917-I96I) me servira à illustrer cette thèse.

Malgré la publication de ses archives, notamment dans les volumes The Legend of Maya Deren, les études contenues dans Maya Deren and the American Avant-Garde, dirigé par Bill Nicoll'3, les remarquables Dialogues théoriques avec Maya Deren d'Alain Alcide-Sudre (1996) et la publication de ses

I. Je me réfère notamment à la réflexion de G. Deleuze sur la catastrophe : «appelons diagramme, à la suite de Bacon, [...] cette double notion - autour de laquelle on tourne depuis le début - de catastrophe germe ou chaos germe», dans "La voix de Gilles Deleuze», cours du 3I/03/8I [en ligne]. Disponible sur <http://www2.univ-paris8.fr/deleuze/article. php3?id_article $=45>$ (consulté le 25/06/2014).

2. M. Deyen, "New Directions in Film Art», dans Essential Deren - Collected Writing on Film by Maya Deren, édition et préface de B. R. McPherson, Kingston, New-York, Documentext, 2005, p. 208. (Désormais abrégé : $E D$ )

3. V. A. Clark, M. Hodson, C. Neiman, The Legend of Maya Deren. A Documentary Biography and Collected Works, New York, Anthology Film Archives - Film Culture, 1984, 1988, 2 vol. 
écrits théoriques en français ${ }^{4}$, son œuvre reste peu connue en France. Opposée à la fois au naturalisme et au surréalisme, elle s'est occupée à définir une poétique filmique autonome de l'image-temps comme créatrice d'une expérience émotionnelle dépassant la subjectivité. Elle se positionne relativement aux productions de l'avant-garde européenne (Cavalcanti, Ruttman, Dziga Vertov, Cocteau, Dali, Man Ray) pour définir un art qui est difficilement reçu en temps de guerre. Les études récentes ont souligné la part essentielle de son mari, le réalisateur d'origine tchèque Alexander Hackenschmied (1907-2004), ou Alexander Hamid, qui initie Maya Deren aux techniques cinématographiques lors du tournage de Meshes of the Afternoon (1943), dans lequel il incarne luimême le jeune homme. Alexander Hammid, qui continue à travailler avec Maya Deren, comme chef opérateur ou technicien, jusqu'en 1946, apporte l'expérience de l'avant-garde cinématographique tchèque et est considéré comme l'un des promoteurs de l'avant-garde cinématographique américaine, bien qu'il soit essentiellement connu pour ses documentaires.

Le spectateur qui découvre aujourd'hui le petit nombre de courts métrages de Maya Deren ne peut qu'être stupéfait par leur modernité. Ils semblent étrangers à leur époque. Sa réflexion théorique anticipe de façon frappante sur les thèmes deleuziens. D'autre part, il est évident qu'elle produit ses films avec une conscience exceptionnelle de leur signification dans l'histoire de la cinématographie, cherchant à se distancer de l'idéologie réaliste du documentaire soutenue par le climat politique de la Seconde Guerre mondiale aux États-Unis et contre le système hollywoodien. Néanmoins, chez cette Antigone du cinéma d'auteur, c'est bien une "poésie de la vision filmiques" qui est en question - elle utilise aussi, comme Man Ray et Benjamin Fondane, le terme de «cine-poems $s^{6}$ » au nom d'une conception ascétique, sévère et exclusive du filmique pur?.

Chez Maya Deren, le filmique est défini de façon fonctionnelle par le fait qu'il entretient avec les autres arts la même relation que la poésie à la littérature. Cela implique une suspension de la narrativité commune à la poésie et au filmique qui le rapproche des structures musicales. Le filmique est d'emblée pensé en termes visuels, et non linguistiques, expliquant sa transition personnelle d'une poésie verbale à un poétique filmique : "Je n'ai pas été une très bonne poétesse parce qu'en réalité mon esprit pensait en images que je tentais

4. M. Deren, Écrits sur l'art et le cinéma, trad. de l'anglais par E. Alloi et J. Beaulieu, Paris, Paris Experimental, 2004.

5. M. Deren, «Film in the Classicist Tradition», brochure publicitaire (fin I946), ED, p. 249.

6. Voir R. Jackson, "The Modernist Poetics of Maya Deren", dans B. Nichols, Maya Deren and the American Avant-garde, Berkeley, California University Press, 200I, p. 5I.

7. M. Deren, "Art as a Moving Picture», Brochure (1960), ED, p. 255. 
de traduire ou de décrire avec des mots ${ }^{8}$.» Au cours de la réalisation de ses films expérimentaux, la spécificité filmique présuppose explicitement une suspension de la structuration verbale de ses contenus pour penser directement le film en termes d'espace-temps : "Le film est art d'espace-temps?.»

Cela présuppose que la cohérence, dite réaliste, de l'espace et du temps, peut être désarticulée pour faire place à une cohérence seconde de type filmique ("cinematic coherence and integrity ${ }^{\mathrm{TO}}$ ) qui se présente en termes de discontinuité, de contraction ou d'extension temporelle. Il y a dislocation du réel ("dislocations of reality ${ }^{\mathrm{II}}$ ") et ce que nous pourrions appeler un processus de désidentification de l'action humaine, individuelle et collective, à l'égard des concepts qui permettrait de subsumer et de définir la nature de l'action : "Aucunes descriptions verbales ne peuvent rendre compte d'un matériau

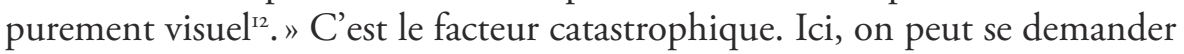
pourquoi la réalisatrice n'a pas exploré plus systématiquement les possibilités d'une bande sonore non verbale pour ses films silencieux, même si la musique japonaise de Teji Ito, ajoutée en 1959 à Meshes of the Afternoon, apporte au film une fonction rythmique, et une sorte de déterritorialisation sonore, qui nous semble être un enrichissement considérable aujourd'hui.

En termes de montage, le mouvement d'un personnage amorcé dans un espace donné peut donc parfaitement être suivi par un plan se déroulant dans un espace différent, comme cela se voit dans les courts métrages Meshes of the Afternoon (1943) et At land (1944). À un certain moment, un mouvement corporel visuellement unique et continu traverse cinq espaces différents juxtaposés en plans successifs ${ }^{13}$ : le sol d'une plage, un sol meuble, une prairie herbeuse, un sol dallé, un sol lisse. C'est une catastrophe dans la représentation de la narrativité. Grâce au montage, la continuité du mouvement corporel sert ici à relier des

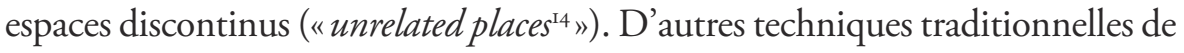
l'avant-garde, l'inversion de la direction de défilement de la pellicule ("reverse motion»), le ralentissement des images, l'usage du négatif, l'arrêt sur image sont aussi considérées comme spécifiquement filmiques, et sont parfois empruntées aux films de Cocteau, notamment au Sang d'un poète (1930' ${ }^{\mathrm{IS}}$. L'espace-temps

8. M. Deren, lettre à James Carr (1955), ED, p. I90-I9I.

9. M. Deren, "Magic is New» (1946), ED, p. 205.

Io. Ibid.

II. M. Deren, "Cinema as an Art Form» (I946), ED, p. 32.

I2. Ibid.

13. At Land, 1944 (I5 min, muet. Photographie: H. Heyman et A. Hammid). Réalisé par M. Deren et A. Hammid. Séquences : IO:I9 à IO:I2.

I4. M. Deren, "Cinema as an Art Form», ED, p. 32.

I5. M. Deren, "Cinematography: The Creative Use of Reality», ED, p. I22. 
spécifique créé par le montage est irréductible à la capture objective des images qui en fournit la matière. Pour Maya Deren, cette transgression du réalisme produit une fluidité ( $f$ luid»; «volatil») filmique qui pourrait être confrontée à nouveau, sous l'éclairage deleuzien, avec les analyses bergsoniennes de Matière et mémoire (1896) et de L'Évolution créatrice (1907) qui lui parviennent, d'après Renata Jackson, à travers sa lecture de The Philosophy of Intensive Manifolds de Thomas Ernest Hulme ${ }^{16}$. Notons que cette conception est aujourd'hui rejointe par Téresa Faucon sous le nom d'une «théorie dynamique» du montage ${ }^{17}$.

Dans ces courts métrages, la continuité causale et narrative de l'intrigue ne sert plus de fil conducteur au montage des plans, opposant le filmique poétique au positionnement verbal performatif opéré par les dialogues des personnages dans un film traditionnel. En général, le travail filmique est toujours affecté d'une fonction transcendantale au sens kantien, ce qui implique un principe d'économie maximale dans la réalisation et la production au lieu d'induire une mise à disposition objective maximale des décors, des personnages etc. Lorsqu'elle filme un danseur dans Ritual in Transfigured Time, l'accélération de ses mouvements appartient à la fonction filmique, et non à la vitesse «normale» du danseur, impossible à obtenir sur une scène dans la réalitée ${ }^{18}$. De même, le ralentissement de la course dans l'escalier de la jeune femme jouée par Maya Deren dans Meshes of the Afternoon dans trois plans successifs. Enfin, comme dans Le Sang d'un poète de Cocteau, les lois physiques, mais aussi les coordonnées phénoménologiques naturelles de la perception, sont transgressées par le montage, comme dans la scène où le personnage se trouve aspiré dans l'espace vertical, flottant dans la chambre, frôlant les plafonds et les murs, et percevant l'espace de la pièce d'un point de vue surplombant. Ainsi, un espace filmique est défini par les mouvements qui se déploient en lui, et non l'inverse : «Pour moi, une grande pièce est une pièce que l'on met longtemps à traverser ${ }^{19}$.» Le corps détermine son espace. C'est sans doute pourquoi Maya Deren a concentré peu à peu ses recherches expérimentales sur la chorégraphie, cherchant à montrer que l'espace filmique est véritablement induit par le corps dansant, ce qui suppose la suppression de son espace matériel traditionnel ${ }^{20}$.

Dans Meshes of the Afternoon, une seconde ascension dans l'escalier nous montre le cadre de référence de la photographie basculant de droite à gauche. Ce qui semble remarquable, c'est que cette fonction transcendantale,

I6. Voir R. Jackson, "The Modernist Poetics of Maya Deren», dans B. Nichols, Maya Deren and the American Avant-Garde, Berkeley, Califormia University Press, 200I, p. 58.

17. T. Faucon, Théorie du montage : énergie, forces et fluides, Paris, Armand Colin, 2013.

18. Ibid., p. 193.

19. M. Deren, "New Directions in Film Art» (195I), ED, p. 2 I4.

20. M. Deren, "Choregraphy for the Camera» (I945), ED, p. 22I. 
constitutive du filmique, n'est coordonnée ni à des impératifs psychologiques, ni à des impératifs théoriques d'ordre philosophique. Il ne s'agit plus d'exprimer quelque chose, mais de penser en cinéma ${ }^{21}$. Il en résulte une conception héraclitéenne du filmique où les identités stables, le principe de contradiction, les propriétés physiques de l'espace et du temps, les disjonctions liées aux simultanéités, bref, l'ordre imposé à la perception par une sensibilité et un entendement intersubjectifs, vont faire place à un univers métamorphique. Ici, on doit noter que si le premier film de Maya Deren et Peter Hamid a concentré l'intérêt des critiques, c'est qu'il rassemble une grande diversité de moyens proprement filmiques, par exemple la transgression du principe des indiscernables, lorsque la protagoniste principale, interprétée par Maya Deren, est multipliée visuellement par deux, puis implicitement par trois dans une scène unique. Le principe d'identité, qu'il soit celui des objets ou celui du sujet pris comme référence par la caméra, explose et confronte immédiatement le spectateur, en retour, au mystère de la constitution de sa propre identité dans le temps.

Ici, il faut certainement s'arrêter au problème central de l'expression dans le film expérimental. Maya Deren réitère fréquemment son refus de penser le film expérimental comme une expression du psychologique conçu comme une collection d'états subjectifs individuels, qui seraient intentionnellement traduits en symboles ou en images, et tels qu'ils pourraient être fournis par une histoire biographique personnelle ${ }^{22}$. De la sorte, la centralité romantique du moi dans l'art est rejetée. L'œuvre n'exprime rien, mais crée un effet. Ainsi elle n'exprime pas un affect, mais créé un affect ("A form which creates pain $\left.{ }^{23} »\right)$. Ainsi, le présupposé naïf ("Que voulez-vous dire?»), validé comme acte d'expression linguistique, doit être déconstruit comme appartenant au métalangage des langues naturelles. Comment faire pour que l'œuvre filmique ne soit pas immédiatement interprétée (avec ses outils narratifs et ses codes de représentation) dans les termes du métalangage naturel? C'est le problème que Maya Deren aborde dans ses conférences de vulgarisation pour faire comprendre la spécificité de ses films dans l'horizon culturel américain des années 1940, alors dominé par le pragmatisme qui se traduit par des finalités sociales de l'œuvre (le divertissement, le perfectionnement des connaissances). Cela est particulièrement vrai dans la période de guerre au cours de laquelle elle a défendu sa conception du poétique, au moment où l'horizon d'attente du spectateur est surdéterminé par une fonction idéologique du documentaire et du reportage de guerre ${ }^{24}$.

2I. Ibid., p. 194.

22. M. Deren, «New Directions in Film Art» (I95I), ED, p. 208-2II.

23. M. Deren, "An Anagram of Ideas on Art, Form and Film» (1946), ED, p. 54.

24. Voir sa tentative de situation du film à l'égard du documentaire dans «Cinema as an Art Form» (1946), ED, p. 27. 
Selon sa conception du film, le rapport d'expression (qui inclut le rapport symbolique) fait place à un jeu d'équivalences entre un événement filmique et un pathos ou une idée philosophique. Ce qui pourrait induire le spectateur en erreur, c'est la réitération dans Meshes of the afternoon d'éléments symboliques classiques (la fleur, la clé, le miroir, le couteau, le personnage en noir) suggérant un déchiffrement analogue à celui du mythe ou de l'allégorie. Or, l'intention de Maya Deren est inverse, bien que, fille d'un psychiatre américain renommé, elle soit très informée des processus analytiques freudiens et des grilles d'interprétation des ouvres d'art par Freud. Cependant, selon elle, le film ne saurait constituer une expression réelle du processus de refoulement et de projections névrotiques pour la simple raison que les éléments imaginatifs du psychisme individuel sont sélectionnés, recomposés et intégrés selon un principe d'organisation extérieur à eux, idée ou émotion, pour advenir à une forme possédant une dynamique autonome ("its reality and meaning are contained within itself and in the dynamics of the inter-relationships of its components parts ${ }^{25} »$ ). Sans nier une valeur symbolique minimale de l'image (qu'elle associe occasionnellement à des archétypes jungiens), elle tient à en réduire au maximum les codifications, observant par exemple que toute bonne peinture de madone peut transmettre un sentiment de dévotion et d'exaltation ${ }^{26}$.

Il est remarquable que ses écrits théoriques se soient concentrés sur le processus de réception de ses œuvres, en soulignant qu'il ne devait pas être analytique, mais au contraire, être un contact immédiat présupposant une réceptivité naïve («innocent receptivity ${ }^{27}$ ») avec l'univers visuel du film. Cela signifie, d'une part, que toute classification théorique, par exemple surréaliste, risque, par son appareil d'analyse, de trahir la spécificité filmique de l'œuvre mais, d'autre part également, que l'horizon phénoménologique d'attente du spectateur, fondé sur les principes d'une objectivité de la représentation sociale, doit nécessairement dénaturer l'œuvre qui lui est proposée. Il est donc impossible d'aborder ce projet sans le replacer dans une réflexion sur l'ontologie, car le poétique déconstruit les codifications sociales de la représentation cinématographique du réel.

En ce sens, Maya Deren poursuit une réflexion amorcée sur le filmique pur ( «le cinéma pur») dans les années I920 et 1930 en Europe (Henri Chomette, Germaine Dulac) avec les films expérimentaux non formalistes, et continuée par des philosophes du cinéma, par exemple Benjamin Fondane ${ }^{28}$. Le refus du

25. "sa réalité et son sens sont limités par son existence propre et par la dynamique interne des éléments qui le constituent» (M. Deren, "Cinema as an Art Form» [1946], ED, p. 25).

26. M. Deren, "An Anagram of Ideas on Art, Form and Film» (I946), ED, p. 9I.

27. M. Deren, "Cinema as an Art Form» (1946), ED, p. 23.

28. B. Fondane, Écrits pour le cinéma, introduction et notes par O. Salazar-Ferrer et R. Fotiade, Lagrasse, Verdier, coll. « Poche», 2007. 
psychologique, des grilles de perception et d'attente induites par une histoire culturelle, mais aussi par toute hétéronomie de l'œuvre, qu'elle soit dépendante d'un système de création artistique, politique, idéologique, esthétique, moral («Une ouvre d'art présuppose l'abandon de tout système personnel [...] un abandon de l'histoire, une innocence par rapport au passé29»). Nous retrouvons ici les grands principes des avant-gardes qui se caractérisent souvent par la décision d'anéantir la pesanteur écrasante du passé. Chez Maya Deren, ce qui nous frappe aujourd'hui, c'est sa lutte contre les principes idéologiques du cinéma commercial au profit d'un cinéma d'auteur, un "cinéma de chambre», contre-pouvoir permanent opposé au totalitarisme de l'industrie cinématographique américaine. Il existe donc une révolution phénoménologique engendrée par le contact avec le poétique, une catastrophe dans les principes de réception d'une œuvre :

[...] l'individu, privé des références absolues qui déterminaient les cadres moraux de sa vie, est obligé de rechercher d'une façon urgente et désespérée une unité suffisamment régulière pour constituer une identité, et suffisamment souple pour lier les éléments d'un univers en apparence anarchique, dont la gravité, les révolutions, les constellations obéissent à une logique qu'il lui reste encore à découvrir ${ }^{30}$.

Cependant, il serait faux de penser que pour Maya Deren, le film bénéficierait d'une autonomie ontologique complète, car il conserve une relation à un universel, en particulier pathétique, pour utiliser un terme de la phénoménologie matérielle de Michel Henry, par exemple l'affect de peur ("fear») qui, selon elle, occupe dans l'économie d'un film expérimental le rôle d'un subjectif universel ("collective subjective ${ }^{3 \mathrm{r}}$ )). Il n'y a donc pas de place pour un filmique purement formel et ludique. Le film abstrait (par exemple Ballet mécanique de Fernand Léger) qu'elle appelle une "peinture animée» ne l'intéresse pas. Car il est hors de question de parvenir à une image non identifiable ("unrecognizable») qui lui ferait perdre sa pertinence (son autorité, sa fidélité et sa réalité phénoménologiques) et, simultanément, ses pouvoirs de suggestion et d'interprétation. Toutefois, pour éviter ce danger, il n'y a de solution qu'à rapporter le filmique ("the cinematic form») à sa capacité à constituer de l'espace-temps avec des images photographiques de constitution réaliste ("the photographic image as reality ${ }^{32}$ ).

29. M. Deren, «New Directions in Film Art» (195I), ED, p. 2II. Elle évoque aussi "an unprejudiced receptivity, free of personal requirements and preconceptions» dans "An Anagram of Ideas, Form and Film", ED, p. 67.

30. M. Deren, "Cinema as an Art Form" (1946), ED, p. 31.

3I. M. Deren, «New Directions in Film Art» (I95I), ED, p. 208.

32. M. Deren, "Cinematography: The Creative Use of Reality» (I960), ED, p. I22 et I26. 
J'en viens à la seconde fonction du poétique qui consiste à créer une forme nouvelle de perception, ou de conscience, si cette forme de perception est réfléchie. Certes, il s'agit d'une totalité autonome ("emergent whole») irréductible à chacune de ses parties dans la tradition de la Gelstalt theorie. Mais il ne s'agit pas seulement d'une forme. Pour Maya Deren, l'œuvre filmique crée un type de manifestation : «En art, le problème est de créer un objet matériel avec une existence sensible ${ }^{33}$.» Elle est consciente, exactement comme Merleau-Ponty dans L'CEil et l'Esprit, que l'approche scientifique de l'objet dépasse ou détruit sa manifestation sensible ${ }^{34}$. La composition filmique, elle, manifeste un "pathos ${ }^{35}$ " - le terme est de Maya Deren - ou bien peut constituer un équivalent d'une idée philosophique comme dans Méditation on violence, mais le poétique réside dans la création d'une nouvelle expérience visuelle ("My purpose in neither to instruct, nor entertain, but to be that experience which is poetry ${ }^{36}$.»). Nous pourrions lier cette approche à l'analyse de l'œuvre d'art comme ce qui nous donne à «sentir ce que nous sommes, cette vie dans l'épreuve de sa passion, de son angoisse, de sa souffrance et de sa joie ${ }^{37}$ ». Dans la phénoménologie matérielle henryenne, en effet, c'est une critique de l'intentionnalité du sujet et de la représentativité de l'image qui la fonde comme auto-affection de la vie par elle-même. Paradoxalement, le rejet de la représentativité symbolique de l'image filmique au profit d'une totalité organique et dynamique de l'œuvre rend possible la compréhension de l'expérience en termes d'immanence. Pourtant, nous le savons, cette expérience crée une "réalité semi-psychologique ${ }^{38}$ " qui nous confronte avec des topos existentiels fondamentaux liés à une prise de conscience de la dimension tragique de la temporalisation de la conscience, qui serait alors le contenu du poétique filmique.

C'est pourquoi, je suis assez perplexe devant l'insistance de Renata Jackson sur le rôle d'une "éthique humaniste» dans la poétique de Maya Deren, à partir des déclarations d'après lesquelles la création artistique éclairerait certains principes éthiques et moraux («illuminate certain moral and ethical principles»). On voit mal comment le travail filmique tel que le pratique Maya Deren pourrait «clarifier les conditions sociales» du réalisateur, dans le cadre d'une

33. M. Deren, «New Directions in Film Art» (1951), ED, p. 209.

34. Ibid.

35. Ibid., p. 213 .

36. «Mon objectif n'est ni d'instruire, ni de divertir, mais de faire l'expérience de la poésie elle-même." (Ibid., p. 219.)

37. M. Henry, «Kandinsky : le mystère des dernières œuvres», dans Phénoménologie de la vie, t. III, De l'Art et du politique, Paris, PUF, coll. «Épiméthée», 2004, p. 225.

38. M. Deren, «An Anagram of Ideas on Art, Form and Film» (1946), ED, p. 35. 
éthique humaniste ${ }^{39}$. Les déclarations de Maya Deren, en situant le travail cinématographique dans l'histoire de la culture moderne, me semblent surtout souligner que l'œuvre filmique doit augmenter ("growth and development in the individual»), par une conscience de soi, la portée des expériences nouvelles qu'elle crée, dans la culture d'une civilisation.

Dans At land (1943), son second film, qu'elle conçoit comme une "Odyssée inversée", la stabilité des continuités spatiales est brisée tandis que le mouvement corporel du personnage principal, une jeune femme seule sur une plage, explore cet espace en métamorphose qui semble indifférent à sa volonté. Néanmoins, des éléments fortement symboliques restent présents : la célèbre scène où la jeune femme grimpe sur un arbre sec sur la plage et parvient sans transition à une longue table d'invités sur laquelle elle va ramper, sans être vue de ceux-ci, traversant des feuillages que le montage a introduits dans la scène, puis le jeu d'échecs dont une pièce va s'échapper et être poursuivie par la jeune femme dans des espaces successifs. Au début des séquences, la réalisatrice utilise une inversion de la pellicule pour montrer les vagues de la mer revenir en arrière et défaire l'ordre du temps. Ces séquences oniriques, soutenues par la performance et la puissance du jeu expressif de Maya Deren comme actrice (que l'on n'a pas assez signalé sous la pression de son rôle de réalisatrice et de théoricienne), constituent bien une unité haptique de l'espace à partir des forces physiologique (le corps, les mains, les pieds nus). En ce sens, l'approche de Maya Deren anticipe clairement sur l'image-mouvement deleuzienne où le mouvement ne s'ajoute pas à une image, mais la constitue comme telle ${ }^{40}$. L'instabilité ontologique de l'espace physique, très onirique, répond à l'expressivité du regard de l'actrice qui en vise avec étonnement et stupéfaction les manifestations sensibles. Les éléments traditionnels, le jeu d'échecs sur la plage devant la mer (qui semble repris par Bergman en 1957 dans une séquence du film Le Septième Sceau), le vol d'une pièce du jeu, et le dédoublement du personnage principal sont certes ouverts à l'interprétation.

Dans son déroulement, le court métrage effectue une transition entre des espaces ouverts (la mer, le ciel, le chemin) et des espaces fermés (la pièce enfumée, l'espace rocheux de la rivière, la cabane). Il donne ainsi à voir un processus d'intériorisation où le temps est sensible à travers sa matérialité physiologique et élémentaire où domine l'eau en mouvement. Lorsque la jeune femme marche sur un chemin et que l'homme qui l'aborde change successivement et insensiblement d'identité, la métaphore existentielle se forme clairement, comme par une contraction temporelle analogue à la vitesse du souvenir. Au niveau

39. R. Jackson, art. cité, p. 55.

40. Voir G. Deleuze, L'Image-mouvement, Paris, Minuit, coll. «Critique», I983. 
méta-cinématographique, la séquence peut apparaître comme une Odyssée métaphysique, inspirée de façon très libre de l'arrivée d'Ulysse sur le rivage des Phéaciens au chant V. Pourtant, l'inversion du temps, au début, indique clairement le début d'une régression du temps. Ce film, s'il fallait le replacer dans une interprétation, offrirait une méditation saisissante sur l'expérience existentielle du temps, voire une reprise émotionnelle et phénoménologique de toute naissance au monde. On sait que Maya Deren l'interprétait comme un contraste entre un moi permanent et un monde volatil sans identités stables.

Si nous tentions un parallèle avec un traitement littéraire du poétique, ce serait avec la poésie de René Char. Le poème «Les premiers instants» (1947), par exemple, offre le même rapport de distanciation à l'égard de la continuité événementielle et l'instabilité des identités ${ }^{41}$. Sous le signe de l'eau créatrice, l'expérience de la temporalité créatrice du narrateur se donne sous une série d'images irréductible à une narrativité, mais dans une contraction extrême du contenu. L'improbabilité des connexions d'images et leur défamiliarisation sont néanmoins ancrées dans des formes référentielles ("Nous regardions couler devant nous l'eau grandissante.»). La séparation de l'objectivité (ce qui est visé par l'activité intentionnelle d'un sujet connaissant) et d'un sujet (celui qui exerce une visée noétique vers un objet quelconque) disparaît tout comme dans Meshes of the Afternoon, les objets ou l'espace objectif s'animent eux-mêmes pour participer à la création de l'espace sensible dans lequel le personnage central évolue : "L'escalier lui-même, de nature inanimée, intervient (de même que les autres objets dans le film) pour gêner les efforts de la jeune fille qui tente d'arriver quelque part ("even an ostensibly inanimate staircase conspired [as do other objects in the film] to frustrate a girl in her effort to arrive somewhere $\left.{ }^{42} »\right)$. Ce qui nous semble certain, c'est que le poétique vise à restituer ou à constituer une forme, selon laquelle la séparation dure entre le subjectif et l'objectif, issue de la révolution galiléenne du XvII ${ }^{\mathrm{e}}$ siècle, est absorbée, et comme résolue, par l'unité d'une synthèse heureuse. Au moment où la crise du parlant est presque oubliée malgré le combat d'un René Clair, d'un Chaplin ou d'un Benjamin Fondane, pour défendre la spécificité du filmique, sonore

4I. «Les premiers instants»: Nous regardions couler devant nous l'eau grandissante. Elle effaçait d'un coup la montagne, se chassant de ses flancs maternels. Ce n'était pas un torrent qui s'offrait à son destin mais une bête ineffable dont nous devenions la parole et la substance. Elle nous tenait amoureux sur l'arc tout-puissant de son imagination. Quelle intervention eût pu nous contraindre? La modicité quotidienne avait fui, le sang jeté était rendu à sa chaleur. Adoptés par l'ouvert, poncés jusqu'à l'invisible, nous étions une victoire qui ne prendrait jamais fin» ("La fontaine narrative», dans Commune présence, préface de G. Blin, Paris, Gallimard, I978, p. I06).

42. M. Deren, «Creating Movies with a New Dimension: Time» (I946), ED, p. I34. 
ou non, se dresse une conception polémique presque anachronique du cinéma aux États-Unis, alimentée par le flux d'idées et de réflexions que l'émigration apporte avec elle. On gagnerait à comparer la conception de Maya Deren avec un inédit récemment publié de Benjamin Fondane intitulé : "Le poème cinématographique» (1929). Lorsque Fondane écrit ce texte, il vient de présenter les films les plus représentatifs du cinéma d'avant-garde en Argentine :

Pour avoir voulu trouver une sortie à la condition humaine, le poème du cinéma a commencé par être vide, par être inhumain; l'homme nous empêche de voir l'homme. Les premiers poèmes purs de Richter, de Ruttmann, étaient complètement abstraits, mouvements purs de volumes, de superficies, de lignes géométriques. Mais le véritable chemin de la recherche exige que nous n'abandonnions pas complètement l'homme, mais que nous brisions ses systèmes de relations, ses jugements sur l'espace, le temps, la loi, la nécessité, pour explorer à travers lui le sentiment de la vie, de cette vie, dont chacun des actes possède quelque chose de miraculeux et de désordonné. Notre vraie misère est de vivre dans le merveilleux sans nous en rendre compte ${ }^{43}$.

Ces remarques nous orientent vers l'idée que le poétique, dans une culture saturée de langage et d'images, a orienté sa puissance de rupture vers une compréhension prérationnelle des grandes angoisses existentielles liées à la temporalité. Lorsqu' elle participa au colloque «Poetry and the Film» en 1953, Maya Deren déclara : «Un poème, à mon sens, crée des formes visibles ou audibles pour quelque chose d'invisible, qui est le sentiment ou l'émotion, ou

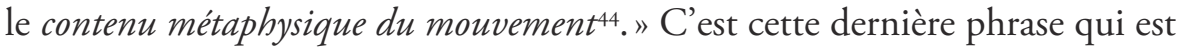
intéressante, en cela qu'elle identifie l'objet central de «l'investigation verticale» qui caractérise le poétique, par opposition au développement horizontal des syntagmes dans une ouvre. Dans les analyses qui suivent, je voudrais développer une réflexion sur l'identification du poétique avec ce «contenu métaphysique du mouvement».

De même que l'image filmique, l'image verbale de René Char, par exemple l'eau grandissante, n'est pas un symbole, ce qui tient lieu d'une autre chose, irréductible à elle, mais l'évocation d'une expérience homogène pathétiquement ou émotionnellement à ce qui doit se dire. On peut supposer que la vision d'une rivière naissante, puis grandissante, s'offre comme intuition du dynamisme continu de la création poétique, homogène au temps subjectif, emportant le poète dans un mouvement qui progresse vers une liberté indéfinie de la vision («l'ouvert») et victorieuse par là même. Les efforts pour rabattre la sémantique du poème sur un substrat d'expériences et de références réelles

43. B. Fondane, «Le poème cinématographique», dans O. Salazar-Ferrer (dir.), «Benjamin Fondane, esthétique, Photographie et cinéma", La Part de l'Cil (Bruxelles), nº 22-23, 2013.

44. Transcription citée par R. Jackson, art. cité, p. 57. 
localisables dans la biographie de son auteur, tels qu'ils furent entrepris par Paul Veyne, me semblent inutiles ${ }^{45}$.

Une autre séquence emblématique de Maya Deren représente une soirée entre amis dans Ritual in Transfigurated Time (1945-1946). Le début du film, montre deux femmes qui font écho à l'image traditionnelle du destin dans la mythologie grecque, avec les Parques Lachesis (interprétée par Maya Deren) et Atropos (qui coupe le fil des destinées). Les déplacements et les gestes des invités, rencontrant d'autres personnes, puis les quittant, sont montés en continuité, avec des mouvements repris de personnage en personnage, de sorte que l'ensemble forme une chorégraphie. La généralisation du mouvement par le montage et le ralentissement des images sont "ritualisés». Derrière la forme dynamique, et l'effet esthétique de cette gestion du temps, c'est le thème angoissant ou tragique de la solitude et de la communauté qui ressort du contraste entre les mouvements individuels et l'unité organique du groupe social. Les couples aussitôt formés se défont et sortent du cadre en visant par leurs regards une autre personne située hors champ. La scène est composée de mouvements circulaires, induits par le mouvement de la caméra mais aussi par les mouvements de rotation des acteurs sur eux-mêmes, qui, par le montage, constituent un tourbillon collectif. Maya Deren a opposé des expressions visuelles de l'espoir à une répétition indéfinie des mouvements circulaires, créant une impression d'angoisse. La répétition des gestes et des expressions de reconnaissance des visages alterne avec les séparations physiques, mais aussi avec les retours en boucle des mêmes séquences qui accélèrent, donnant au spectateur l'effet d'une chorégraphie tragique.

La poétique filmique de Maya Deren nous renvoie brutalement à un stade antérieur aux dissociations fictives de la rationalité, tout en nous confrontant à des équivalents visuels nous permettant de rejouer les émotions et les affects liés aux grands thèmes existentiels (solitude et communauté, contingence et nécessité, objectivité et subjectivité, mémoire et finitude, différence et répétition), en les inscrivant dans la production sans fin de formes par la vie. C'est pourquoi le terme de "métaphysique», utilisé surtout dans les dernières années, est justifiét ${ }^{6}$. L'indépendance de cette poétique filmique qui refuse de s'inscrire dans une théorie de type surréaliste ou psychanalytique, explique sa vitalité théorique.

Maya Deren aime traiter ses films techniquement, en mettant complètement entre parenthèses ses sources psychologiques. Le métadiscours objectif,

45. P. Veyne, René Char en ses poèmes, Paris, Gallimard, coll. "Nrf Essais», I990.

46. M. Deren, "The Very Eye of Night», "Statement of Purpose» (1959), Film Culture, n 29, I963, ED, p. 231. 
technique, qu'elle développe, à la façon des théoriciens du Bauhaus, est résolument impersonnel. Un contresens fréquent consiste à rattacher cet univers filmique au surréalisme alors que son concept de «ritualisation» est explicitement opposé "aux extases du confessionnal surréaliste ${ }^{47}$ » qui présuppose à la fois l'illusion d'une connaissance et une exaltation de soi préjudiciables au poétique conçu comme une forme dramatique transcendant la personnalité individuelle. L'œuvre filmique n'est pas une connaissance de l'inconscient ou d'autre chose (la fonction épistémologique est réservée aux sciences expérimentales), mais la production volontaire d'un rituel visuel. Le rituel est avant tout une pratique sociale dépersonnalisée, qui implique des formes narratives abstraites, souvent mythiques, visant à agir ou à communiquer avec une divinité qui reste, en tant que telle, inconnue et toute-puissante. Maya Deren y retrouve l'autonomie du poétique filmique, mais aussi un tragique figuré ("the tragic figure $\left.^{48} »\right)$. Si ses composantes religieuses ne sont que suggérées, sa dimension performative est essentielle : «la ritualisation filmique est une production consciente d'une nouvelle réalité, un instrument qui permet de comprendre et de manipuler un univers où l'homme doit se situer d'une façon ou d'une autre ${ }^{49}$ ». Si nous nous arrêtons là, le poétique filmique est simplement "une forme de vie» dans laquelle seront joués dynamiquement des affects ( $a$ new set of relationships affects ${ }^{5 \circ}$ ») chez le spectateur.

Comme dans L'Année dernière à Marienbad (196I) d'Alain Resnais, les scènes semblent acquérir une ontologie propre, séparée de la convention habituelle qui associe le spectateur et le réalisateur par un pacte fictif de réalité. Comme le remarque Thomas Beltzer :

Marienbad pose l'existence séparée de ses personnages. Du point de vue cinématographique, c'est l'étude d'une réalité séparée de sa propre existence, évitant les conventions du réalisme comme fausse illusion. Par sa propre discontinuité temporelle, ses personnages sans nom et son décor hermétiquement clos, il exige que nous l'acceptions comme une réalité en elle-même plutôt qu'une fidèle et en dernière analyse, illusoire représentation du réel. Marienbad nous dit que, par sa construction, l'art est une réalité ajoutée à la réalité et non une copie de la réalitést.

L'essai théorique le plus achevé qui cherche à définir les positions esthétiques de Maya Deren dans l'histoire de la culture et des sciences de son temps est "An Anagram of Ideas on Art, Form and Film» (1946), qu’ elle rédige significativement

47. M. Deren, "An Anagram of Ideas on Art, Form and Film» (1946), ED, p. 57.

48. Ibid., p. 59.

49. Ibid.

50. Ibid., p. 64.

5I. T. Beltzer, «Last Year at Marienbad: An Intertextual Meditation», Senses of cinema, $n^{\circ}$ IO, novembre 2000. 
après le tournage de Ritual. Rejetant d'un côté la tendance naturaliste et de l'autre la tendance surréaliste, mais aussi un romantisme plaçant le sujet psychologique au centre de l'expression, Maya Deren insiste sur l'abandon de l'expressivité (communication, transmission ou recréation d'une expérience) comme fonction artistique, s'opposant de plein fouet au système hollywoodien. Elle repousse systématiquement l'idée d'une poétique surréaliste. En réalité, marquée par le New Criticism, la poésie de T.-S. Eliot et l'imagisme d'Ezra Pound, elle avait consacré son mémoire de maîtrise à "L'influence de l'école symboliste française sur la poésie anglo-américaine». Grande admiratrice de l'esthétique de Paul Valéry qu'elle aborde en français, Maya Deren se comprend elle-même, de façon paradoxale, comme un auteur classique ${ }^{{ }^{2}}$. Elle est particulièrement sensible à la préface fondamentale de Paul Valéry à Adonis de La Fontaine, qu'il vaut la peine de citer :

Qui dit exactitude et style, invoque le contraire du songe; et qui les rencontre dans un ouvrage doit supposer dans son auteur toute la peine et tout le temps qu'il lui fallut pour s'opposer à la dissipation permanente des pensées. Les plus belles, comme les autres, toutes, ce sont des ombres : et les fantômes, ici, précèdent les vivants. Ce ne fut jamais un jeu d'oisif que de soustraire un peu de grâce, un peu de clarté, un peu de durée, à la mobilité des choses de l'esprit : et que de changer ce qui passe en ce qui subsiste. Et plus la proie que l'on convoite est-elle inquiète et fugitive, plus faut-il de présence et de volonté pour la rendre éternellement présente, dans son attitude éternellement fuyante ${ }^{\varsigma_{3}}$.

Je suis certain que ces lignes marquèrent Maya Deren, par l'incidence inattendue qu'elles pouvaient apporter sur une compréhension du cinéma comme synthèse de la fugacité et de l'éternité. La solution est l'image-temps. Ainsi, Ritual est une ouvre classique, essentiellement parce que ce film cherche à transcender ses sources individuelles et subjectives dans une forme ritualisée. Dans les films de Deren, la temporalité et l'espace ne sont jamais traités de façon euclidienne comme des milieux homogènes, mais comme un matériau, par exemple dans l'inversion de la pellicule ("Not only reveals a new quality

52. "I would like to use the word "classicist" to describe Ritual in Transfigurated Time precisely because it does not define according to the elements of content-factual, fictional, abstract or psychological. It is a concept of a method: a controlled manipulation of any or all elements into a form which will transcend and transfigure them." ("Je voudrais utiliser le mot "classique" pour décrire Rituel dans un temps transfiguré à cause justement du fait qu'il n'implique pas des éléments du contenu - qu'ils soient factuels, fictifs, abstraits ou psychologiques, mais le concept d'une méthode : celle d'une manipulation contrôlée d'un élément quelconque ou de tous les éléments en une forme qui pourra les transcender et les transfigurer." " Ritual in Transfigurated Time» [1946], ED, p. 228.) La même thèse est défendue dans un court manifeste : «Films in the Classicist Tradition", ED, p. 250.

53. P. Valéry, introduction à, La Fontaine, Adonis, Devambez, Au masque d'or, coll. «Le Florilège français", I92I, p. IV. 
in the motion of the waves, but creates, to put it mildly, almost revolutionary reality ${ }^{54}$ »). Par exemple, l'effet visé de la réversibilité n'est pas un effet burlesque, comme c'est le cas dans le film comique muet américain, mais consiste à suggérer visuellement une réversibilité de l'ordre des événements dans le temps filmique. En ce sens, le travail sur l'espace est toujours déterminé par un travail sur la temporalité. Dans la scène de la soirée, les arrêts sur image ou le ralentissement de la vitesse des images s'inscrivant dans un montage à vitesse normale introduisent une dissociation qualitative dans les temporalités, ce qui sera utilisé plus tard par Wong Kar Waiss. Les mouvements rapides du visage de Maya Deren déroulant une bobine de fil autour de ses bras dans Ritual, tandis qu'elle chante, sont ralentis de sorte que les effets plastiques de la vitesse réelle du sujet, par exemple le mouvement des cheveux, sont donnés dans une visibilité spécifiquement filmique, inaccessible à l'œil en réalités ${ }^{6}$.

Au début de la séquence de la soirée, nous apercevons deux arrêts sur image, très brefs, qui poignardent le spectateur, montrant les invités dans une pétrification soudaine de leurs mouvements. Et nous ne sommes pas en face de n'importe quels mouvements : les invités vont s'embrasser dans un seul mouvement d'ensemble et ils se figent soudain dans la glace du temps. Leurs mains vont se joindre au moment où ils sont arrêtés par cet instant meurtrier. Certes, au même moment, la jeune femme veuve en noir vient d'entrer. Elle vient de parvenir au bout d'une bobine de fil, enroulé par une autre femme qui vient de disparaître, laissant une chaise vide. Est-ce la mort qui, phénoménologiquement, s'exprime dans ces deux arrêts sur images? Certainement, en l'absence de langage, la réalisatrice a décidé de traduire en image-mouvement ce qui n'est pas verbalisé. Ces arrêts ne peuvent qu'être reçus comme des indices d'impossibilités, nous mettant en présence du tragique et de l'angoisse inhérents à la temporalité qui nous est présentée comme tourbillon, juste après, avec des vitesses variables. La répétition de scènes identiques, à peu de distance, sur la pellicule, anticipe sur les innovations techniques d'Alain Resnais, dans L'Année dernière à Marienbad, malgré les propos très sévères prononcés contre son film ${ }^{57}$.

Il y a dans ces répétitions internes un équivalent visuel du mouvement effectué par la reprise kierkegaardienne, métamorphose conçue comme "élévation en puissance» qui consiste à «exprimer l'éternel dans la succession de la

54. M. Deren, "An Anagram of Ideas on Art, Form and Film» (1946), ED, p. IO3.

55. A. Coppola, Le cinéma asiatique : Chine, Corée, Japon, Hong-Kong, Taïwan, Paris, L'Harmattan, 2004, p. I64.

56. M. Deren, "An Anagram of Ideas on Art, Form and Film» (1946), ED, p. IO2.

57. Voir M. Deren, "Some Metaphors for the Creative Process", The Village Voice, 25 août I960", ED, p. 242. 
temporalité» où le souvenir éclaire l'interprétation nouvelle du passés ${ }^{8}$. Le film lui-même pourrait être considéré comme une reprise au sens kierkegaardien, par une décision qui constituerait peut-être l'essence du poétique au cinéma. Je veux dire que la méditation sur la temporalité ne ressort pas, ici, comme dans le cinéma hollywoodien, de la mort du héros, à la suite de facteurs objectifs, qu'ils soient constitués par une lutte hérö̈que, une fatalité sociale ou un sacrifice moral. Ici, la tragédie temporelle est existentielle et apparaît comme manifestation filmique. La plasticité de l'image-temps fonctionne comme une exploration de ses possibilités, mais ce travail est intégré à une réflexion obsessionnelle sur la temporalité que j'appellerais «tragique».

Pour tenter d'aller plus loin, j'avancerai une interprétation extérieure aux écrits de Deren, qui reste fondamentalement davantage préoccupée de décrire l'autonomie de l'univers filmique que d'analyser ses interprétations métafilmiques. Toutefois, je cherche à expliciter le lien du filmique comme rituel au tragique, déjà évoqué. De quel tragique s'agit-il? Mon impression est que l'autonomie du filmique, la ritualisation, fondée sur un travail sur la temporalité, ne peut qu'être perçue comme angoisse fondamentale de la temporalité par le spectateur. Maya Deren, en utilisant le terme de "métaphysique» à la fin de sa vie, en était d'ailleurs conscientes9.

Les films de Maya Deren les plus impressionnants, notamment Meshes of the Afternoon et Ritual in Transfigurated Time, nous apparaissent intimement liés à une poétique du temps, c'est-à-dire à une «spectographie» qui offre au spectateur une matérialisation visuelle et haptique de sa plasticité, et le met en contact émotionnel intense avec la déréalisation ontologique, c'est-à-dire avec la fragilité de la manifestation visuelle du monde. La ritualisation, chez Maya Deren, caractérise des passages critiques, c'est-à-dire des métamorphoses, thèse qu'elle emprunte certainement à l'anthropologie américaine, sans doute à Margaret $\mathrm{Mead}^{60}$. Ainsi, la fin de Ritual in Transfigurated Time nous montre le passage à la projection de la pellicule négative, moment où la jeune veuve devient blanche. Cette blancheur est obtenue par un procédé filmique qui n'emprunte rien à l'objectivité de la couleur. De telles métamorphoses sont liées au temps de façon intrinsèque, c'est-à-dire qu'elles ne se déroulent pas dans le temps conçu comme un milieu vide et homogène, mais dans un temps qui est inhérent à leur mode filmique : «Étant un film rituel, il est réalisé non

58. J'utilise ici les termes de l'introduction de N. Viallanex à S. Kierkegaard, La Répétition, trad. N. Viallanex, Paris, Flammarion, coll. "GF», 1990, p. 22.

59. M. Deren, "Art as a Moving Picture», Brochure (1960), ED, p. 255.

6o. Voir M. Mead, Coming of Age in Samoa - A Study of Adolescence and Sex in Primitive Societies (1928), Londres, Penguin Book, 1969. 
seulement en termes spatiaux, mais en termes de Temps créé par la caméra ${ }^{6 \mathrm{I}}$.» Or ce travail sur la matière temps a justement pour conséquence d'affecter la sensibilité par une réflexion sur sa propre temporalité, par sa nature même, en tant qu'elle est reçue comme modalité filmique. C'est bien pourquoi, le terme "tragique» revient chez Deren malgré tous les efforts qu'elle effectue pour souligner l'autonomie formelle du filmique.

On peut objecter que les procédés de l'œuvre filmique, en réalité, si nous considérons l'ensemble des courts-métrages, visent un ensemble plus large de grands motifs existentiels : la continuité du subjectif et la volatilité du monde extérieur, la multiplication de la subjectivité, le renouvellement de la subjectivité par elle-même, etc. Pourtant, le motif central, éminemment bergsonien, reste la temporalisation du monde dans la conscience, et nous pourrions ajouter, dans la conscience cinématographique. D'autre part, si l'on accepte que le fait que celle qui réalise le film offre son apparence à l'un des personnages centraux comme dans Ritual in Transfigurated Time, l'image devient autoréférentielle et peut viser le travail sur l'image-temps effectué par Maya Deren (dans le rôle de Lachesis), à la fois sur elle-même, et sur les amis qui sont les acteurs de ses films. De même, lorsque ses amis tiennent la caméra (par exemple Alexander Hamid ou Hella Heyman dans At land), ils participent eux-mêmes à cette temporalisation tragique qui les lie à une éternité visuelle non réaliste. Le cinéma, ici, pourrait bien n'avoir d'autre objet que lui-même au sens où son travail sur l'image-temps éveille en nous la tragédie inhérente à toute temporalité? Voilà quelle peut être l'interprétation de l'affirmation selon laquelle le poétique porte sur le «contenu métaphysique du mouvement». Le film n'est-il pas répétition dans l'irréversibilité du monde?

Cette interprétation n'est pas celle que la réalisatrice propose de ses films, toujours méfiante envers les risques de toute interprétation théorique, ou même philosophique, mais comme dans Le Testament d'Orphée, elle ressort obligatoirement de la coïncidence entre les fonctions multiples du film de l'auteurréalisateur et photographe-acteur qui se donne les moyens d'entrer lui-même dans l'espace filmique qu'il crée. Comme dans le roman La Invención de Morel (1940) de Bioy Casares, où le protagoniste parvient sur une île où se déclenche chaque soir dans une villa luxueuse la séquence holographique d'une soirée sans cesse réitérée à l'identique comme un film en trois dimensions, mais qui n'a pu être «tourné» qu'en tuant par son processus ses acteurs involontaires. Dans ce roman, le héros, amoureux d'une femme (Faustina) appartenant à cette cohorte de fantômes, va pénétrer le mécanisme qui a permis de créer ce film holographique, et y entrer, avant de constater qu'il va mourir. Malgré le

6I. M. Deren, «Ritual in Transfigurated Time» (1946), ED, 227. 
style de Casares, souvent pesant, l'idée centrale permet de penser les paradoxes de l'acte de la création filmique.

La ritualisation chez Maya Deren me semble donc inséparable de la valeur de reprise, au sens kierkegaardien. L'œuvre filmique nous confronte avec une visualisation de la reprise, mais devient elle-même objet d'une reprise lorsqu'elle est visionnée successivement par le spectateur. Le film, identique objectivement à lui-même, est ressaisi différemment à chaque fois qu'il est subjectivement revu. Dans les écrits théoriques de Maya Deren, il est très peu question d'esthétique. Le mot "beauté" est absent de ses analyses sur l'art filmique. Comme Benjamin Fondane dans le Faux Traité d'esthétique (1938), elle vise essentiellement une autonomie du poétique à l'égard de l'Histoire, mais aussi des finalités contraignantes, de nature sociale ou culturelle, dans un système lié à des intérêts économiques, idéologiques et politiques. En s'établissant toujours comme fonction catastrophique, le poétique filmique, par ses moyens spécifiques, nous confronte à chaque fois au mystère de la temporalisation du moi, en particulier dans Ritual in Transfigurated Time. Le poétique du filmique, conçu comme rituel, tient à cette reprise, «à la seconde puissance», de la durée, qui en ressaisit les motifs existentiels fondamentaux. 